\title{
Avaliação da retenção hídrica com a técnica do soro fisiológico em suspensão durante a artroscopia do ombro*
}

\author{
Evaluation of fluid retention when using saline \\ in suspension during shoulder arthroscopy
}

\author{
Alexandre Almeida ${ }^{1}$, Eduardo StanguerlinI ${ }^{2}$, GILberto Roveda ${ }^{3}$, \\ MÁrcio Rangel Valin ${ }^{4}$, NAYvaldo Couto de Almeida ${ }^{5}$
}

\section{RESUMO}

Objetivo: Avaliar a retenção hídrica após a artroscopia do ombro quando utilizada a técnica de infusão de soro fisiológico em suspensão, com o propósito de identificar os pacientes com risco para ganho hídrico excessivo no transoperatório. Métodos: Foi analisado um grupo de 131 pacientes, após uma única exclusão, que foram submetidos à artroscopia do ombro e tiveram seu índice de massa corpo-

* Trabalho realizado no Hospital Saúde de Caxias do Sul (RS), Brasil.

1. Médico Ortopedista do Hospital Saúde de Caxias do Sul (RS), Brasil.

2. Médico Ortopedista do Hospital Saúde de Caxias do Sul (RS), Brasil.

3. Médico Ortopedista do Hospital Saúde de Caxias do Sul (RS), Brasil.

4. Médico Ortopedista do Hospital Saúde de Caxias do Sul (RS), Brasil.

5. Médico Ortopedista do Hospital Saúde de Caxias do Sul (RS), Brasil.

Endereço para correspondência: Rua Vitório Buzelatto, 222/601, Bairro Madureira - 95020-290 - Caxias do Sul (RS), Brasil. Tel.: 55-54-3221-4163. E-mail: ale@ visao.com.br

Recebido em 23/7/07. Aprovado para publicação em 30/10/07. Copyright RBO2007 ral (IMC) aferido para fins de classificação entre os diferentes graus de obesidade. $O$ posicionamento foi o decúbito lateral com o membro superior em suporte de tração. A distensão articular utilizou o soro fisiológico em suspensão com equipo de quatro vias e quatro frascos de soro fisiológico de $2.000 \mathrm{ml}$ colocados na mesma altura, deixando a pressão de infusão variar entre 31 e $97 \mathrm{mmHg}$. Os pacientes foram submetidos à medição do peso tão logo tinham condições de ficar em posição ortostática ao lado da cama na sala de recuperação. As variáveis estudadas foram: peso corporal pré e pósoperatório, IMC, quantidade de soro fisiológico utilizado no sistema de irrigação, quantidade de soro fisiológico infundido endovenoso e o tempo do procedimento cirúrgico. Resultados: $\mathrm{O}$ ganho hídrico médio aferido na série de pacientes avaliados foi de 1.901 ( \pm 968) gramas. Foi encontrada correlação moderada entre o soro fisiológico do sistema de irrigação e o ganho hídrico $\left(\mathrm{r}=0,372 ; \mathrm{r}^{2}=0,138 ; \mathrm{P}<\right.$ $0,001)$, bem como para o aporte endovenoso e $o$ ganho hídrico $\left(r=0,253 ; r^{2}=0,064 ; P=0,004\right)$. Quando os aportes hídricos foram verificados em conjunto, aparece correlação significativa, mas de efeito moderado $\left(r=0,381 ; r^{2}=0,0146 ; P<0,001\right)$. Foi encontrada correlação de magnitude maior entre o tempo do ato cirúrgico e o ganho hídrico 
apresentado pelos pacientes $\left(r=0,586 ; r^{2}=0,264\right.$; $P<0,001)$. Ao agrupar os pacientes com IMC abaixo de 30 (normal e sobrepeso) e compará-los com os com IMC acima de 30 (obesidade grau 1, 2 e mórbida), foi verificada a existência de retenção hídrica maior entre os obesos $(2.111 \mathrm{~g} \pm 1.144 \mathrm{~g})$ (mediana de $1.900 \mathrm{~g})$ do que nos não obesos $(1.851 \mathrm{~g} \pm 921 \mathrm{~g})$ (mediana: 1.754g) $(P=\mathbf{0 , 2 9 2})$. Conclusões: A retenção hídrica encontrada foi de $1.901( \pm 968)$ gramas. Apenas 14,6\% do ganho de peso encontrado são decorrentes do aporte hídrico transoperatório. Pacientes submetidos a procedimentos artroscópicos prolongados retêm mais líquido. Pacientes com obesidade graus 1, 2 e mórbida parecem ter mais expressiva tendência para maior ganho de peso transoperatório.

Descritores - Articulação do ombro/cirurgia; Artroscopia; Obesidade; Índice de massa corporal; Irrigação/métodos; Estudos prospectivos

\section{ABSTRACT}

Objective: To evaluate fluid retention after shoulder arthroscopy using infusion of saline in suspension in order to identify those patients at risk for excessive fluid gain during surgery. Methods: After only one exclusion a group of 131 patients submitted to shoulder arthroscopy were studied and had their Bodily Mass Index (BMI) assessed for the purpose of obesity classification. The patients were positioned laying on their side with the upper limb on a traction support. Joint distension was performed using saline in suspension with a four-way line and four $2000 \mathrm{ml}$ saline flasks placed at the same height, and infusion pressure varied between 31 and 97 mmHg. Patients were weighted as soon as they were able to reach an orthostatic position by the bedside in the recovery room. Variables studied were pre- and post-operative bodily weight, BMI, amount of saline used in the irrigation system, amount of saline used in intravenous infusion, and length of surgery. Results: The average fluid gain in this series of patients was 1901 ( \pm 968) grams. A moderate correlation was found between saline from the irrigation system and fluid gain ( $\left.r=0.372 ; r^{2}=0.138 ; P<0.001\right)$, and between intravenous intake and fluid gain $(r=0,253$; $\left.r^{2}=0.064 ; P=0.004\right)$. When saline intakes were considered as a whole, there is a significant correlation, but with a moderate effect ( $r=0.381 ; r^{2}$ $=0.0146 ; P<0.001)$. A higher magnitude correlation was found between surgery length and fluid gain of the patients $\left(r=0.586 ; r^{2}=0.264 ; P<0.001\right)$. When patients with BMI below 30 (normal and overweight) were grouped together and compared to patients with BMI above 30 (obesity grade 1, 2, and morbid), higher fluid retention was found among obese patients (2111 $\mathrm{g} \pm 1144 \mathrm{~g}$ ) (mean $1900 \mathrm{~g}$ ) than among non-obese patients $(1851 \mathrm{~g} \pm 921 \mathrm{~g})$ (mean $1754 \mathrm{~g})(P=0.292)$. Conclusions: The fluid retention found amounted to 1901 ( \pm 968) grams. Only 14.6\% of the weight gain found is a result of fluid intake during surgery. Patients submitted to lengthy arthroscopic procedures retain more fluid. Patients with Grade 1, 2, and morbid obesity seem to have a more expressive tendency to gain more weight during surgery.

Keywords - Shoulder joint/surgery; Arthroscopy; Obesity; Body mass index; Irrigation/methods; Prospective studies

\section{INTRODUÇÃO}

A artroscopia do ombro é procedimento realizado em meio líquido, que gera uma série de variáveis que devem ser manejadas pelo cirurgião ortopedista: o cuidado com o isolamento do paciente na mesa cirúrgica, a utilização de cautérios de radiofreqüência, o controle da pressão e do fluxo dos líquidos administrados ao paciente e a consequiente retenção do líquido levando a edema local ${ }^{(1)}$. O edema resultante da retenção hídrica local, por sua vez, pode levar a situações como o aumento da dificuldade para realizar o procedimento até complicações graves.

A retenção de líquido pelo organismo, bem como a elevação da pressão que ocorre no músculo deltóide ao final do procedimento, é considerada achado normal e rapidamente revertido nas primeiras horas do pós-operatório ${ }^{(1-2)}$. Lo et al observaram que o edema tenso costuma ser revertido nos primeiros 30 a $45 \mathrm{mi}$ - 
nutos após o procedimento ${ }^{(3)}$. Estes mesmos autores, em seu estudo de coorte, observaram o ganho ponderal em 53 pacientes após a artroscopia do ombro quando utilizada a bomba de pressão articular mantendo a pressão de $60 \mathrm{mmHg}$. Esses autores observaram ganho de peso significativo, porém consideraram o achado um efeito colateral e não uma complicação do procedimento $^{(3)}$. No Hospital Saúde de Caxias do Sul/Rs, utilizamos para irrigação da articulação o soro fisiológico suspenso sob ação gravitacional e anteriormente publicamos nossos achados a respeito da segurança do método ${ }^{(4)}$.

O objetivo desta pesquisa é avaliar a retenção hídrica pelo organismo de um indivíduo submetido à artroscopia do ombro quando utilizada a técnica de infusão de soro fisiológico em suspensão, identificando as características do paciente com risco para ganho de peso excessivo no transoperatório, quando avaliados o tempo cirúrgico e o grau de obesidade segundo o índice de massa corporal.

\section{MÉTODOS}

O estudo realizado foi prospectivo e transversal.

Foi analisado um grupo de 131 pacientes submetidos à artroscopia do ombro no período de 22 de dezembro de 2005 a 29 de maio de 2007.

Todo paciente estudado recebeu um Termo de Consentimento informado aprovado pela Comissão de Ética do Hospital Saúde de Caxias do Sul/Rs, instituição onde o trabalho foi realizado.

Os pacientes foram submetidos à medição da altura e do peso no pré-operatório imediato, para cálculo do índice de massa corporal (IMC). As medidas foram realizadas pelo mesmo observador e através do mesmo instrumental, o paciente vestindo apenas roupa própria para uso em centro cirúrgico.

O IMC $\left(\mathrm{kg} / \mathrm{m}^{2}\right)$ foi utilizado para classificar os pacientes de acordo com o grau de obesidade conforme o quadro $1^{(5)}$.

Depois de realizada a anestesia, o paciente foi posicionado em decúbito lateral com o membro superior (MS) em suporte com tração de $5 \mathrm{~kg}$. A técnica de distensão articular foi o soro fisiológico em suspensão utilizando o equipo da marca Zammi de quatro vias,

\begin{tabular}{|c|c|}
\hline QUADRO 1 & Graus de obesidade \\
\hline Cla & $\begin{array}{l}\text { óo da obesidade } \\
\left(\mathrm{kg} / \mathrm{m}^{2}\right)\end{array}$ \\
\hline $\begin{array}{l}18-24,9 \\
25-29,9 \\
30-34,9 \\
35-39,9 \\
\geq 40,0\end{array}$ & $\begin{array}{l}\text { Normal } \\
\text { Sobrepeso } \\
\text { Obesidade G1 } \\
\text { Obesidade G2 } \\
\text { Obesidade mórbida }\end{array}$ \\
\hline
\end{tabular}

Legenda: IMC - índice de massa corporal, $\mathrm{kg} / \mathrm{m}^{2}$ - unidade de medida do índice de massa corporal.

Fonte: Mancini MC. Noções fundamentais - diagnóstico e classificação da obesidade. In: Garrido Jr AB, Ferraz EM, Barroso FC, Marchesini JB, Szego T. Cirurgia da obesidade. São Paulo: Atheneu; 2002. p. 1-7.

com diâmetro interno de $8 \mathrm{~mm}$. O suporte utilizado permite a colocação dos quatro frascos de soro fisiológico de $2.000 \mathrm{ml}$ na mesma altura $^{(4)}$.

A variação de altura do suporte de soro ficou entre $450 \mathrm{~mm}$ da altura onde se encontrava o ombro do paciente e $1.350 \mathrm{~mm}$, altura limite do pé direito da sala cirúrgica, deixando a pressão de infusão variar entre 31 e $97 \mathrm{mmHg}$, conforme avaliado no estudo de Almeida et $\mathrm{l}^{(4)}$.

Todos os pacientes foram imobilizados, ainda anestesiados na sala cirúrgica, com uma tipóia. Foi utilizado um coxim de abdução quando a lesão do manguito rotador suturada era grande ou extensa. A tipóia, bem como o coxim de abdução, teve seu peso aferido quando utilizada, a fim de ser descontado do cálculo do peso final.

Os pacientes foram submetidos à medição do peso tão logo tinham condições de ficar em posição ortostática ao lado da cama na sala de recuperação.

A quantidade de soro fisiológico utilizada no sistema de irrigação, bem como a quantidade de soro fisiológico infundida endovenosamente, durante o procedimento e durante o período na sala de recuperação até o momento da medida do peso no pós-operatório, foi aferida em $\mathrm{ml}$. O tempo de procedimento cirúrgico foi anotado.

Foi excluído um único paciente portador de insuficiência renal crônica e dependente de diálise, com indicação de receber um mínimo de aporte hídrico endovenoso. 
As variáveis estudadas foram: peso corporal pré e pós-operatório, IMC, quantidade de soro fisiológico utilizado no sistema de irrigação, quantidade de soro fisiológico infundido endovenoso e o tempo do procedimento cirúrgico.

Os dados foram analisados com o programa estatístico SPSS (Statistical Package for Social Sciences) versão 12.0 (sPSS Inc., 1989-2003). Para a análise estatística, foram utilizados: cálculo das médias, desvio-padrão, mediana, freqüência e percentual. Utilizou-se o teste $t$ de Student para comparar as médias das variáveis simétricas e o coeficiente de correlação (r) de Pearson. O teste não paramétrico de Mann-Whitney foi utilizado para análise das variáveis assimétricas. Para avaliar a magnitude das diferenças obtidas, foi utilizada a escala de magnitude adaptada de Will Hopkins. Foram consideradas significantes as diferenças com $p \leq$ 0,05 para um intervalo de confiança de $95 \%$.

O estudo avaliou 130 pacientes com média de idade de 53,9 ( $\pm 12,7)$ anos - idade mínima de 19 anos e máxima de 83 anos.

Com relação ao sexo, 44 pacientes $(33,8 \%)$ eram masculinos e 86 (66,2\%), femininos.

Com relação ao grau de obesidade, 53 pacientes $(40,8 \%)$ apresentavam peso considerado como normal, 52 tinham sobrepeso (40\%), 21 foram classificados como obesos grau $1(16,2 \%)$, dois como obesos grau 2 $(1,5 \%)$ e dois sofrendo obesidade mórbida $(1,5 \%)$.

$\mathrm{O}$ volume médio utilizado para infusão hídrica através do sistema de irrigação artroscópica foi de $13.769 \mathrm{ml}( \pm 7.947 \mathrm{ml})$, com um mínimo de $1.350 \mathrm{ml}$ e um máximo de $38.750 \mathrm{ml}$.

O volume médio utilizado durante a infusão endovenosa foi de $1.164 \mathrm{ml}( \pm 405 \mathrm{ml})$, com o mínimo de $200 \mathrm{ml}$ e o máximo de $2.600 \mathrm{ml}$.

O tempo cirúrgico médio foi de $63,9 \mathrm{~min}( \pm 24,5$ $\mathrm{min}$ ), com a mediana de 61,5 min (IIQ: 45 a 82,3 min), enquanto o tempo médio de intervalo entre o final do ato cirúrgico e a medição do peso no pós-operatório foi de 208,5 $\mathrm{min}( \pm 55,4 \mathrm{~min})$.

$\mathrm{O}$ ganho de peso médio aferido na série de pacientes avaliados foi de 1.901 gramas $( \pm 968 \mathrm{~g})$, com a mediana de 1.789 gramas (IIQ: 1.282 a $2.669 \mathrm{~g}$ ).

\section{RESULTADOS}

Foi encontrada correlação significativa com magnitude de efeito moderada entre o soro fisiológico do sistema de irrigação e o ganho de peso no pós-operatório $\left(\mathrm{r}=0.372 ; \mathrm{r}^{2}=0,138 ; \mathrm{P}<0,001\right)$. Resultado semelhante foi verificado entre o aporte endovenoso e o ganho de peso no pós-operatório, mas com magnitude do efeito pequena $\left(\mathrm{r}=0,253 ; \mathrm{r}^{2}=0,064 ; \mathrm{P}=0,004\right)$. Quando os aportes hídricos foram analisados em conjunto, identificou-se correlação significativa, mas de efeito moderado $\left(\mathrm{r}=0,381 ; \mathrm{r}^{2}=0,0146 ; \mathrm{P}<0,001\right)$. Essa relação sugere que $14,6 \%$ do ganho de peso se devem ao aporte combinado. Foi encontrada correlação de magnitude maior entre o tempo do ato cirúrgico e o ganho de peso pós-operatório apresentado pelos pacientes $\left(r=0,586 ; r^{2}=0,264 ; P<0,001\right)$.

Não houve diferença estatística entre o ganho de peso pós-operatório e o IMC $\left(\mathrm{r}=0,055 ; \mathrm{r}^{2}=0,004 ; \mathrm{P}=0,535\right)$. Ao agrupar os pacientes com IMC abaixo de 30 (normal e sobrepeso) e compará-los com os pacientes com IMC acima de 30 (obesidade graus 1, 2 e mórbida), foi verificada a existência de ganho de peso maior entre os obesos (média: $2.111 \mathrm{~g} \pm 1.144 \mathrm{~g}$ ) (mediana: $1.900 \mathrm{~g}$ ) do que nos não obesos (média: $1.851 \mathrm{~g} \pm 921 \mathrm{~g}$ ) (mediana: $1.754 \mathrm{~g}$ ). A diferença encontrada pelo teste de Mann-Whitney não foi significativa $(\mathrm{P}=0,292)$.

\section{DISCUSSÃO}

O soro fisiológico utilizado habitualmente na artroscopia do ombro é uma solução isotônica. Essa solução é particularmente importante na clínica médica, pois pode ser infundida no sangue sem qualquer perigo de perturbar os equilíbrios osmóticos entre os líquidos intra e extracelulares ${ }^{(6)}$. Se essa solução salina isotônica é acrescentada ao compartimento líquido extracelular, a osmolalidade do líquido extracelular não se modifica e não haverá osmose. O único efeito é o aumento do volume do líquido extracelular ${ }^{(6)}$.

Quando uma quantidade extra de líquido se acumula no espaço extracelular, a pressão intersticial se eleva de seu valor negativo normal (subatmosférico) para um valor positivo, para fazer com que os tecidos sofram tumefação tão intensa que se tornam, então, capazes de conter até 10 a 30 litros de líquido extracelu- 
lar. Isto é, os espaços intersticiais passam a constituir literalmente um reservatório de transbordamento para o excesso de líquido ${ }^{(7)}$. Esse eficiente mecanismo de compensação faz com que esse volume de líquido seja absorvido lentamente pelo organismo e excretado para o exterior sem gerar aumento da pré-carga ${ }^{(2,6-9)}$.

A idéia de que o aporte de soro fisiológico durante a artroscopia do ombro é inócua fica corroborada na literatura devido ao baixo índice de complicações diretamente relacionadas ao fato ${ }^{(10)}$. No entanto, quando o sistema de compensação corporal falha, as complicações podem ocorrer, sendo geralmente graves e preocupam a equipe cirúrgica. São citadas: lesões neurológicas ${ }^{(11-14)}$, necrose de pele ${ }^{(15)}$, sobrecarga hídrica ${ }^{(10)}$, obstrução da via aérea ${ }^{(16-17)}$ e comprometimento da capacidade ventilatória por excessivo extravasamento de líquido ${ }^{(18-19)}$.

$\mathrm{Na}$ procura de fatores que possam contribuir para o perfil do paciente com maior risco de apresentar retenção hídrica excessiva, estudamos a obesidade, por se tratar de um dos principais problemas de saúde pública da sociedade moderna ${ }^{(5,20)}$. Obesidade acarreta risco aumentado de inúmeras doenças crônicas, como diabete melito, dislipidemia, doença cárdio e cerebrovascular, alterações da coagulação, doenças articulares degenerativas, neoplasias estrogênio-dependentes, neoplasia de vesícula biliar, esteatose hepática com ou sem cirrose, apnéia do sono, etc. ${ }^{(5,21-22)}$. Dentre as patologias, a insuficiência renal crônica e a insuficiência cardíaca congestiva, por se tratar de condições que alteram o sistema regulador de líquidos do organismo, são de particular interesse ao cirurgião de ombro ${ }^{(6,23)}$.

A avaliação da obesidade pode ser feita de várias maneiras. Dentre elas, o IMC, também conhecido por índice de Quetelet, é o mais utilizado. Sua obtenção é feita através de um cálculo simples dividindo o peso, em quilogramas, pelo quadrado da altura, em metros. Ele apresenta boa correlação com a adiposidade corporal; no entanto, tem como limitações o fato de não distinguir entre gordura central e periférica e tampouco entre massa gordurosa de massa magra, podendo superestimar o grau de obesidade em indivíduos musculosos ${ }^{(5,24)}$.

Lo et al, avaliando o ganho de peso de pacientes em artroscopia do ombro, verificaram que os fatores que aumentavam o tempo cirúrgico, como a maior comple- xidade do procedimento, levavam ao conseqüente aumento do ganho ponderal pós-operatório ${ }^{(3)}$. Em nosso estudo, procuramos avaliar a magnitude das diferenças obtidas através da escala de magnitude adaptada de Will Hopkins e também definimos o tempo cirúrgico como o principal responsável na gênese do ganho de peso pósoperatório. A escala demonstrou ser o tempo cirúrgico mais importante do que ambos os aportes hídricos fornecidos ao paciente.

Ao ser analisado o perfil do paciente segundo o IMC, foi verificada maior tendência para ganho de peso pósoperatório em pacientes classificados em obesidade grau 1, obesidade grau 2 e obesidade mórbida, muito embora essa diferença não tenha sido significativa ( $\mathrm{P}$ $=0,292$ ). Não foram encontrados trabalhos que fizessem alusão ao perfil do paciente de risco para ganho de peso após procedimento artroscópico.

Acreditamos que o número de pacientes e a diversidade de procedimentos intra e extra-articulares possam ter interferido no ganho de peso pós-operatório no grupo estudado.

O tempo cirúrgico apresentou variação ampla e talvez tenha comprometido a análise da amostra. Acreditamos que uma nova pesquisa, com número maior de pacientes e tempos cirúrgicos semelhantes, nos permita comprovar se a tendência de maior ganho ponderal em obesos é verdadeira.

\section{CONCLUSÕES}

O ganho de peso de um indivíduo submetido à artroscopia do ombro pela técnica de infusão de soro fisiológico em suspensão foi de $1.901( \pm 968)$ gramas. A análise estatística demonstrou que apenas 14,6\% do ganho de peso encontrado são decorrentes do aporte hídrico transoperatório.

Ao analisar o perfil do paciente com risco de ganho ponderal excessivo, foi verificado que aqueles submetidos a procedimentos artroscópicos prolongados retêm mais líquido. Os pacientes com obesidade graus 1, 2 e mórbida parecem ter mais expressiva tendência para maior ganho de peso após a artroscopia do ombro e, portanto, em sua avaliação pré-operatória, deve haver atenção para investigação de afecções cardiovasculares e renais. 


\section{REFERÊNCIAS}

1. Weber SC, Abrams JS, Nottage WM. Complications associated with arthroscopic shoulder surgery. Arthroscopy. 2002;18(2 Suppl 1):88-95.

2. Lee YF, Cohn L, Tooke SM. Intramuscular deltoid pressure during shoulder arthroscopy. Arthroscopy. 1989;5(3):209-12.

3. Lo IK, Burkhart SS. Immediate postoperative fluid retention and weight gain after shoulder arthroscopy. Arthroscopy. 2005; 21(5):605-10.

4. Almeida A, Agostini AP, Valin MR, Martins JA, Ferreira R. Artroscopia do ombro com infusão de soro fisiológico em suspensão. Estamos trabalhando de forma segura? Rev Bras Ortop. 2006;41(7):253-8.

5. Mancini MC. Noções fundamentais - diagnóstico e classificação da obesidade. In: Garrido Jr AB, Ferraz EM, Barroso FC, Marchesini JB, Szego T. Cirurgia da obesidade. São Paulo: Atheneu; 2002. p.1-7.

6. Divisão dos líquidos corporais: equilíbrios osmóticos entre os líquidos extracelulares e intracelulares. In: Guyton AC. Tratado de fisiologia médica. 7a ed. Rio de Janeiro: Guanabara Koogan; 1986. p. 307-5

7. Regulação do volume sanguíneo, do volume de líquido extracelular. In: Guyton AC. Tratado de fisiologia médica. 7a ed. Rio de Janeiro: Guanabara Koogan; 1986. p. 340-9.

8. Ogilvie-Harris DJ, Boynton E. Arthroscopic acromioplasty: extravasation of fluid into the deltoid muscle. Arthroscopy. 1990;6(1):52-4.

9. Carr CF, Murphy JM. Deltoid and supraspinatus muscle pressures following various arthroscopic shoulder procedures. Arthroscopy. 1995;11(4):401-3.

10. McFarland EG, O'Neill OR, Hsu CY. Complications of shoulder arthroscopy. J South Orthop Assoc. 1997;6(3):190-6.

11. Shaffer BS, Tibone JE. Arthroscopic shoulder instability surgery. Complications. Clin Sports Med. 1999;18(4):737-67.

12. Rodeo SA, Forster RA, Weiland AJ. Neurological complications due to arthroscopy. J Bone Joint Surg Am. 1993; 75(6):917-26.
13. Stanish WD, Peterson DC. Shoulder arthroscopy and nerve injury: pitfalls and prevention. Arthroscopy. 1995;11(4):45866.

14. Berjano P, González BG, Olmedo JF, Perez-España LA, Munilla MG. Complications in arthroscopic shoulder surgery. Arthroscopy. 1998;14(8):785-8.

15. Mohammed KD, Hayes MG, Saies AD. Unusual complications of shoulder arthroscopy. J Shoulder Elbow Surg. 2000;9(4): 350-3.

16. Borgeat A, Bird P, Ekatodramis G, Dumont C. Tracheal compression caused by periarticular fluid accumulation: a rare complication of shoulder surgery. J Shoulder Elbow Surg. 2000;9(5):443-5.

17. Hynson M, Tung A, Guevara JE, Katz JA, Glick JM, Shapiro WA. Complete airway obstruction during arthroscopic shoulder surgery. Anesth Analg. 1993;76(4):875-8.

18. Lau KY. Pneumomediastinum caused by subcutaneous emphysema in the shoulder. A rare complication of arthroscopy. Chest. 1993;103(5):1606-7.

19. Lee HC, Dewan N, Crosby L. Subcutaneous emphysema, pneumomediastinum, and potentially life-threatening tension pneumothorax. Pulmonary complications from arthroscopic shoulder decompression. Chest. 1992;101(5):1265-7.

20. Seidell JC. Epidemiology: definition and classification of obesity. In: Kopelman PG, Stock MJ, editors. Clinical obesity. Oxford: Blackwell Science; 1998. p.1-17.

21. Austin H, Austin JM Jr, Partridge EE, Hatch KD, Shingleton HM. Endometrial cancer, obesity, and body fat distribution. Cancer Res. 1991;51(2):568-72.

22. Sims EA, Berchtold P. Obesity and hypertension. Mechanisms and implications for management. JAMA. 1982;247(1):49-52.

23. Lopes Júnior C, Romanek RM, Miranda MM, Posso IP. Anestesia e analgesia pós-operatória em obesos mórbidos. In: Garrido Jr AB, Ferraz EM, Barroso FC, Marchesini JB, Szego T. Cirurgia da obesidade. São Paulo: Atheneu; 2002. p.91-103.

24. Bray GA. Complications of obesity. Ann Intern Med. 1985; 103(6 Pt 2): 1052-62. 